\title{
Entern, kentern oder auflaufen? Zu den Aussichten der Piratenpartei im deutschen Parteiensystem
}

\author{
Holger Onken und Sebastian H. Schneider
}

Nach ihrer Gründung im September 2006 konnte die Piratenpartei zunächst nur in sehr begrenztem Umfang Stimmen gewinnen. Bei den ersten drei Landtagswahlen, an denen sie zwischen Anfang 2008 und Januar 2009 teilnahm $^{1}$, mobilisierte sie zwischen 0,2 und 0,5 Prozent der Wähler. Es hatte zunächst den Anschein, als würde es sich um eine weitere Partei handeln, die dauerhaft den „Sonstigen“ zugeordnet werden könne. Mit einem Ergebnis von 0,9 Prozent bei der Europawahl im Juni 2009 gelang den Piraten ein erster Achtungserfolg. Eine weitere Aufmerksamkeitsschwelle überwanden sie bei der Bundestagswahl im September 2009, als sie mit 2,0 Prozent das beste Ergebnis unter den Kleinstparteien erzielten. Spätestens mit dem Einzug in das Berliner Abgeordnetenhaus im September 2011, als die Partei 8,9 Prozent der abgegebenen Stimmen gewann, stellt sich die Frage, ob sich die Piraten auf der Bundesebene längerfristig etablieren können.

Für einen nachhaltigen Erfolg benötigen Parteien ein Mindestmaß an Verankerung in der Bevölkerung. Dafür müssen Sie als „politische Agenturen“ bestimmte gesellschaftliche Interessen organisieren, die wiederum auf spezifische sozialstrukturell bedingte Lebenslagen, darauf aufbauende Mentalitäten und politische Einstellungen bestimmter Bevölkerungssegmente zurückzuführen sind. Die Festigung der Wählerbasis von Parteien in der Gesamtbevölkerung ergibt sich daraus, dass die politischen Einstellungen und Mentalitäten dieser Basis stabiler sind als ihre Positionen zu einzelnen Sachfragen. ${ }^{2}$ Um die Chancen der Piraten auf nachhaltige Wahlerfolge einschätzen zu können, muss also gefragt werden, ob ihr Erfolg bei der Wahl zum Berliner Abgeordnetenhaus und bei den Landtagswahlen 2012 im Saarland, in Schleswig-Holstein und Nordrhein-Westfalen sowie der anschließende Anstieg der Partei in den Umfragen zur Bundestagswahl auf deutlich mehr als fünf Prozent ${ }^{3}$ auf ein entsprechendes gesellschaftliches Fundament schließen lässt. Die analytische Perspektive dieses Beitrages ist also auf „die Partei“ als „Ausdruck sozialer Kräfte sowie ideologischer und/oder programmatischer Ziele und Forderungen“ "gerichtet. ${ }^{4}$

1 In Hamburg 0,2 Prozent und in Hessen 0,3 beziehungsweise 0,5 Prozent, als es im Januar 2009 nach einem Jahr zu Neuwahlen kam. Für eine Teilnahme an den Landtagswahlen, die in diesem Zeitraum im Bayern und Niedersachsen stattfanden, hatte die Piratenpartei nicht genügend Unterstützungsunterschriften erhalten.

2 Vgl. Thomas von Winter, Politische Orientierungen und Sozialstruktur. Ein Beitrag zur Theorie des Wählerverhaltens, Frankfurt am Main 1987, S. 60 f.

3 Zwischen dem 13. Juli und dem 1. August 2012 wurde durch die Umfrageinstitute Infratest Dimap, Emnid, Forsa und die Forschungsgruppe Wahlen für die Piraten bei der Sonntagsfrage ein Mittelwert von 7,8 Prozent ermittelt.

4 Vgl. Winfried Steffani, Parteien als soziale Organisationen. Zur politologischen Parteienanalyse, in: ZParl, 19. Jg. (1988), H. 4, S. 549 - 560, S. 551 f. 


\section{Entstehung von Parteien auf der Grundlage gesellschaftlicher Konflikte}

Durch historisch-soziologische Analysen wurde nachgewiesen, dass die Genese von Parteien auf fundamentale gesellschaftliche Konflikte zurückzuführen ist. ${ }^{5}$ Solche als „Cleavages“ bezeichneten Konflikte sind demnach entwicklungsgeschichtlich in der gesellschaftlichen Tiefenstruktur verankert. Entlang dieser sozialen und kulturellen Spaltungen bildeten sich in der Demokratisierungsphase seit der zweiten Hälfte des 19. Jahrhunderts in den westeuropäischen Staaten langfristige Koalitionen zwischen sozialen Gruppen, Klassen, Milieus und politischen Parteien ${ }^{6}$, die häufig über Generationen hinweg stabil blieben. Der innere politische Zusammenhalt bestimmter Bevölkerungssegmente, die durch solche Spaltungen von anderen getrennt waren, ergab sich aus geteilten normativen Auffassungen und Wertvorstellungen, die sich wiederum aus einer kulturellen Überformung aufgrund einer gemeinsamen Lebensweise ergaben. ${ }^{7}$ Diese sozialen Gruppen hatten in bedeutenden Fragen weitgehend gemeinsame Vorstellungen davon, wie die Gesellschaft organisiert sein sollte. Die Parteien entwickelten Ideologien und programmatische Konzepte, die ihren Wählern eine Realisierung dieser Vorstellungen versprachen. ${ }^{8}$

Aus der historischen Perspektive wiesen Bevölkerungsgruppen, die ihre Stimme weit überproportional für eine bestimmte Partei abgaben, entweder sozialstrukturell oder kulturell ein hohes Maß an Homogenität auf. Faktisch gehen aus der Zugehörigkeit zu einer bestimmten sozialen Schicht häufig auch spezifische kulturelle Normen hervor. ${ }^{9}$ Folglich gehört zu einem Cleavage immer ein kultureller Aspekt; ein schichtspezifischer gehört häufig ebenfalls dazu, ist jedoch nicht für alle parteibildenden Konflikte eine notwendige Bedingung. Bei der Wählerschaft sozialistischer beziehungsweise sozialdemokratischer Parteien, die die Arbeiterschaft repräsentierten, stand die soziale Schichtung jedoch im Vordergrund. In etwas geringerem Umfang galt das auch für die meisten liberalen Parteien, die einen überproportionalen Stimmenanteil in den Mittelschichten und den höheren Schichten gewannen. Das Bindeglied der Wählerschaften von Parteien, für die die Religion oder die Konfession im Vordergrund steht, ist dagegen kultureller Natur. Das gilt auch für Parteien, die überwiegend sprachliche und kulturelle Minderheiten repräsentieren. Ihre Wählerschaft war hinsichtlich der sozialen Schichtung meist heterogener. ${ }^{10}$

5 Vgl. Seymour Martin Lipset / Stein Rokkan, Cleavage Structures, Party Systems and Voter Alignments: An Introduction, in: dies. (Hrsg.), Party Systems and Voter Alignments, New York 1967, S. 1 - 64. Die Entstehung und Entwicklung von Parteien unterliegt jedoch keinem soziologischen Determinismus. Sie ist abhängig von den Institutionen des politischen Systems, insbesondere der Ausgestaltung des Wahlrechts und dem Verhalten der politischen Eliten beziehungsweise der Bereitschaft der Bürger, persönliche Ressourcen (insbesondere Zeit und Geld) in eine neue Partei einzubringen.

6 Vgl. Franz Urban Pappi, Sozialstruktur, gesellschaftliche Wertorientierungen und Wahlabsicht, in: PVS, 18. Jg. (1977), H. 2, S. 195 - 213.

7 Vgl. Karl Rohe, Wahlen und Wählertraditionen in Deutschland, Frankfurt am Main 1992, S. 24.

8 Vgl. Klaus von Beyme, Parteien in westlichen Demokratien, München 1982, S. 43 ff.

9 Vgl. Gerd Mielke, Gesellschaftliche Konflikte und ihre Repräsentation im deutschen Parteiensystem. Anmerkungen zum Cleavage-Modell von Lipset und Rokkan, in: Ulrich Eith / ders. (Hrsg.), Gesellschaftliche Konflikte und Parteiensysteme, Wiesbaden 2001, S. 77 - 95.

10 Vgl. Stefano Bartolini / Peter Mair, Identity, Competition, and Electoral Availability, New York 1990, S. 213 f. 
Der theoretische Ansatz der auf Stein Rokkan und Seymour Martin Lipset zurückgeht, wurde von der Wahl- und Parteienforschung erstens herangezogen, um die lang anhaltende Stabilität der meisten westeuropäischen Parteiensysteme im 20. Jahrhundert zu erklären. ${ }^{11}$ Zweitens wurden die späten 1960er Jahre als Bezugspunkt identifiziert, um die zunehmende Instabilität vieler westeuropäischer Parteiensysteme seit den $1970 \mathrm{er} \mathrm{Jahren} \mathrm{darzulegen.}{ }^{12}$ In beiden Fällen orientierte sich die Argumentation eng an den historischen Kategorien, die für die Stabilität beziehungsweise Instabilität der Cleavages von Bedeutung waren. Die konzeptionelle Überlegung, dass Parteien gesellschaftliche Konflikte organisieren und die Entstehung neuer Parteien neue Konfliktlagen in der Gesellschaft zum Ausdruck bringt, kann für die Untersuchung ihrer Erfolgsaussichten von hohem heuristischen Nutzen sein. ${ }^{13}$ In der Geschichte der Bundesrepublik gab es verschiedene Fälle, die geeignet sind, diesen $\mathrm{Zu}$ sammenhang darzustellen. Sie zeigen, dass sozialer Wandel und politische Großereignisse zu neuen gesellschaftlichen Konfliktlagen führen können, die den Einzug neuer Parteien in den Bundestag begünstigen.

\section{Erfolge neuer Parteien in der Bundesrepublik Deutschland}

Der am weitesten zurückliegende Fall ist der heute nur noch wenig bekannte „Bund der Heimatvertriebenen und Entrechteten“ (BHE), der sich als „politische Interessenagentur“ der Flüchtlinge aus den östlichen Gebieten des ehemaligen Deutschen Reiches verstand. Die Vertriebenen standen in einem sozialen und wirtschaftlichen Konflikt mit der angestammten Bevölkerung. Der hohe Stimmenanteil des BHE, der bei der Bundestagswahl 1953 mit 5,9 Prozent in den Bundestag einzog, war maßgeblich auf die schlechte wirtschaftliche Lage und die problematische Wohnsituation der Vertriebenen zurückzuführen. ${ }^{14}$ Aufgrund des wirtschaftlichen Erfolges der Bundesrepublik gelang die Integration dieser Bevölkerungsgruppe in den Arbeitsmarkt und die Gesellschaft zunehmend besser. Damit wurde die Wählerbasis des BHE praktisch ausgetrocknet. 1957 verfehlte die Partei den Wiedereinzug in den Bundestag.

Längerfristigere Auswirkungen auf das deutsche Parteiensystem hatte die Entstehung der Grünen in der zweiten Hälfte der 1970er Jahre und ihr Einzug in den Bundestag 1983. Diese Entwicklung wird auf einen Konflikt zwischen materialistischen und postmaterialistischen Werten zurückgeführt, der auf einem gesellschaftlichen Modernisierungsprozess beruht, dessen Grundlage wiederum der über Jahrzehnte zunehmende gesellschaftliche Wohlstand war. Der Ausbau des Sozialstaates, des öffentlichen Beschäftigungssektors und die Bildungsexpansion bewirkten einen postindustriellen Wertewandel, der die Herausbildung sozialer Gruppen förderte, die die (Wähler-)Basis der Grünen bildeten. ${ }^{15}$ Ihre Wert-

11 Vgl. ebenda.

12 Vgl. Russell J. Dalton, The Decline of Party Identifications, in: ders. / Martin P. Wattenberg (Hrsg.), Parties without Partisans, Oxford / New York 2002, S. 19 - 36.

13 Vgl. Stein Rokkan, Towards a Generalized Concept of Verzuiling, in: Political Studies, 24. Jg. (1977), H. 4, S. 563 - 570.

14 Vgl. Werner Kaltefleiter, Politik und Wirtschaft in Deutschland. Konjunktur als Bestimmungsfaktor des Parteiensystems, Köln / Opladen 1968, S. $124 \mathrm{f}$.

15 Vgl. Jens Alber, Modernisierung, neue Spannungslinien und die politischen Chancen der Grünen, in: PVS, 26. Jg. (1985), H. 3, S. $211-226$. 
vorstellungen, so die Deutung, standen im Konflikt mit der materialistischen Orientierung der Mehrheitsgesellschaft. Hinzu komme der Konflikt zwischen libertären und autoritären gesellschaftlichen Werten, die einerseits von den Grünen, andererseits von rechtsextremen Kleinstparteien repräsentiert würden. ${ }^{16}$

Der Wahlerfolg der PDS in den 1990er Jahren ist zunächst als Sonderfall anzusehen, der sich aus den Umständen der deutschen Wiedervereinigung ergeben hat. Er lässt sich dennoch in die Betrachtung, dass Parteien aus gesellschaftlichen Konflikten hervorgehen, einordnen. Die Basis für den PDS-Erfolg bildet ein kulturell-ideologischer Konflikt, in dem Teile der ostdeutschen Bevölkerung den in Westdeutschland dominanten politischen Vorstellungen und gesellschaftlichen Normen ablehnend gegenüber standen. Diese Frontstellung zwischen der PDS und allen anderen Parteien wird entsprechend auch als ZentrumPeripherie-Konflikt charakterisiert ${ }^{17}$, der nach wie vor in deutlich überdurchschnittlichen Ergebnissen der PDS-Nachfolgepartei „Die Linke“ in Ostdeutschland seinen Ausdruck findet. Ihr relativ später Erfolg in den westdeutschen Bundesländern kann ebenfalls auf die politischen Auswirkungen eines politisch-gesellschaftlichen Konfliktes zurückgeführt werden. ${ }^{18}$ Die Partei konnte im Zuge des Strukturwandels des Arbeitsmarktes, der Agenda 2010 und einer Zunahme prekärer Beschäftigungsverhältnisse in begrenztem Umfang reüssieren. ${ }^{19}$

Die angeführten Beispiele haben gemeinsam, dass gesellschaftliche Veränderungen nicht innerhalb des bestehenden Parteiensystems aufgefangen werden konnten. In allen Fällen konnten sich die neuen Parteien auf ein sozialstrukturell, also schichtspezifisch und/oder kulturell-ideologisch abgrenzbares Bevölkerungssegment als Kernwählerschaft stützen und dieses mit ihrem programmatischen Angebot politisch binden. ${ }^{20}$ Die Herausbildung neuer sozialer Gruppen ist auf gesellschaftlichen Wandel zurückzuführen und führt häufig zum Konflikt mit etablierten Gruppen. Die Frage ist dann, ob und wie sich solche Konflikte politisch kanalisieren, ob sie von bestehenden Parteien aufgenommen werden oder eine neue Partei gegründet wird. Ein Weg, um die Erfolgsaussichten der Piratenpartei zu analysieren ist demnach die Untersuchung, ob ein gesellschaftlicher Konflikt existiert, der ihre Entstehung begründen kann: Gibt es ein ausreichend großes Bevölkerungssegment, das eine sozialstrukturelle und/oder kulturelle beziehungsweise mentalitätsbezogene Homogenität auf-

16 Vgl. Gero Neugebauer / Richard Stöss, Die PDS. Geschichte, Organisation, Wähler, Konkurrenten, Opladen 1996, S. 270.

17 Vgl. Oskar Niedermayer, Die Entwicklung des deutschen Parteiensystems: eine empirische Analyse, in: Markus Klein / Wolfgang Jagodzinski / Ekkehard Mochmann / Dieter Ohr (Hrsg.), 50 Jahre Empirische Wahlforschung in Deutschland, Wiesbaden 2000, S. 106 - 125, S. 120.

18 Die PDS erzielte in den westdeutschen Bundesländern bei den vier Bundestagswahlen zwischen 1990 und 2002 Ergebnisse zwischen 0,3 Prozent (1990) und 1,2 Prozent (1998) der abgegebenen Stimmen. Die Partei Die Linke erzielte 2005 4,9 und 2009 8,3 Prozent.

19 Vgl. Manuela Kulick / Holger Onken, Die Wähler der Linkspartei in ihren Hochburgen: Eine empirische Analyse zur niedersächsischen Landtagswahl 2008 in Oldenburg, Delmenhorst und Wilhelmshaven, in: ZParl, 39. Jg. (2008), H. 2, S. 299 - 312. In den westdeutschen Bundesländern findet die Partei bei Wählern in prekären Beschäftigungslagen und unter Arbeitslosen relativ hohen Zuspruch; vgl. Oliver Nachtwey / Tim Spier, Günstige Gelegenheit? Die sozialen und politischen Entstehungshintergründe der Linkspartei, in: Tim Spier / Felix Butzlaff/ Matthias Micus / Franz Walter (Hrsg.), Die Linkspartei: zeitgemäße Idee oder Bündnis ohne Zukunft?, Wiesbaden 2007, S. $13-69$.

20 Der Fall des BHE macht deutlich, dass gesellschaftliche Konflikte, wenn sie gelöst werden, auch politisch irrelevant werden können. 
weist, die zu einem hohen Grad an gemeinsamen Interessen führt, die entsprechend politisierbar sind?

\section{Entwicklung und politische Verortung der Piraten: Hinweise auf ein neues Cleavage?}

Christoph Bieber hat die Entwicklung der Piratenpartei in Deutschland seit ihrer Gründung im September 2006 in vier Phasen eingeteilt, die sich im Hinblick auf die Dynamik unterscheiden, mit der sich die Wahlergebnisse und die Mitgliederzahlen der Partei entwickelt haben. ${ }^{21}$ Mit dem Erfolg bei der Wahl zum Berliner Abgeordnetenhaus im September 2011 sind die Piraten demnach in eine neue Expansionsphase eingetreten. Neben einem Anstieg in den Umfragen ist auch die Anzahl der Parteimitglieder, die seit Januar 2010 stabil bei 12.000 gelegen hatte, innerhalb von nur vier Monaten auf 20.000 angestiegen. ${ }^{22}$

Bei sieben Landtagswahlen, die zwischen dem Achtungserfolg bei der Bundestagswahl und dem Einzug in das Berliner Abgeordnetenhaus stattfanden, erzielte die Piratenpartei Ergebnisse zwischen 1,4 und 2,1 Prozent der abgegebenen Stimmen. Bemerkenswert ist dabei, dass dieser Stimmenanteil in sehr unterschiedlich strukturierten Bundesländern relativ ausgeglichen war: in den westdeutschen Flächenländern Nordrhein-Westfalen (Mai 2010: 1,6 Prozent), Baden-Württemberg und Rheinland-Pfalz (beide März 2011: 2,1 beziehungsweise 1,6 Prozent), in den ostdeutschen Flächenländern Sachsen-Anhalt (März 2011: 1,4 Prozent) und Mecklenburg-Vorpommern (September 2011: 1,9 Prozent) und in den Stadtstaaten Hamburg (Februar 2011: 2,1 Prozent) und Bremen (Mai 2011: 1,9 Prozent). Die naheliegende Vermutung, der Erfolg der Piraten hänge insbesondere mit den für sie günstigen Bedingungen im großstädtischen Milieu zusammen, muss daher nicht unbedingt richtig sein.

Der Impuls für die Gründung der Piratenpartei war von einem Personenkreis ausgegangen, der über keine oder fast keine politischen Erfahrungen verfügte. Dem Gründungsmythos nach sind die Lockerung des Urheberrechts und die Freiheit im Internet von zentraler Bedeutung für die Entstehung der Piraten. ${ }^{23}$ Die Aufmerksamkeit einer breiteren Öffentlichkeit erlangte die Partei erstmals im Zuge der Auseinandersetzung um das Zugangserschwerungsgesetz, das im Zusammenhang mit Internetsperren zur (vermeintlichen) Bekämpfung von Kinderpornographie implementiert wurde. Das Gesetz galt vielen besonders aktiven Internetnutzern als Einfallstor für eine Internetzensur und löste eine an den Petitionsausschuss des Bundestages gerichtete Online-Petition aus, die von mehr als 134.000 Personen unterstützt wurde. Die damit verbundene Kampagne „Löschen statt Sperren“ wurde zwar nicht von der Piratenpartei initiiert, sie konnte aber davon profitieren. Aufgrund ihres Entstehungshintergrundes und der kurzen Entwicklungsphase lag es nahe, die Piraten

21 Nach einer längeren Gründungsphase mit mehreren hundert Mitgliedern (September 2006 bis Juni 2009) trat die Partei in eine viermonatige Wachstumsphase und danach in eine einjährige Stagnations- und Stabilisierungsphase ein. Die jüngste Phase hat demnach mit dem Erfolg bei der Wahl zum Berliner Abgeordnetenhaus begonnen. Vgl. Christoph Bieber, Die Piratenpartei als neue Akteurin im Parteiensystem, in: APuZ, 62. Jg. (2012), H. 7, S. 27 - 32, S. 28 f.

22 Vgl. Piratenwiki, Mitglieder, https://wiki.piratenpartei.de/Mitglieder\#Mitgliederentwicklung (Abruf am 7. August 2012).

23 Zur Gründung der Piratenpartei vgl. Henning Bartels, Die Piratenpartei. Entstehung, Forderungen und Perspektiven der Bewegung, Berlin 2009, S. 57 ff. 
auf eine reine Internetpartei zu reduzieren. ${ }^{24}$ Die Auseinandersetzungen um einzelne Fragen der Netzpolitik könnten jedoch kaum die parteibildende Wirkung eines Cleavage erzeugen, das eine Partei über die Fünfprozenthürde des deutschen Wahlsystems heben würde. Zudem ist eine Einordnung der gegensätzlichen Auffassungen um die Netzpolitik in den Konflikt zwischen einem libertären und einem autoritären Wertesystem, der bereits strukturierende Wirkung im deutschen Parteiensystem entfaltet ${ }^{25}$, plausibel. Den libertären Pol haben demnach die Grünen, den autoritären die rechtsextreme NPD und die Republikaner besetzt. ${ }^{26}$

Eine weitergehende Deutung sieht in den Piraten mehr als eine Netz- und Internetpartei. Der Begriff einer „Freiheitspartei der Informationsgesellschaft“ ${ }^{27}$ könnte auf tiefer greifende gesellschaftliche Veränderungen verweisen, die eine Basis für weitere Erfolge bilden könnten. In dieses Bild passen das Organisationsprinzip und die Partizipationsmöglichkeiten bei den Piraten, durch die das schnelle Mitgliederwachstum begünstigt wurde. Als Beispiele sind die Möglichkeiten einer direkten Beteiligung an Programmdebatten mit Hilfe von „Liquid Feedback " ${ }^{28}$ und Parteitage, auf denen alle Mitglieder stimmberechtigt sind, zu erwähnen. Damit könnten die Piraten eine gesellschaftliche Modernisierung und den Versuch einer Demokratisierung symbolisieren. Hinzu kommt das Kokettieren mit dem eigenen „Amateurstatus“, mit dem man sich einer vermeintlichen „Überprofessionalisierung“ und der unverbindlichen Sprache der etablierten Parteien entgegen stellt. ${ }^{29}$

Abseits der Kernkompetenz der Piraten in der Netzpolitik und bei Urheberrechtsfragen zeichnen sich bisher bei nur wenigen Themen verbindliche programmatische Konturen ab. Dazu gehören die Forderung nach einer Freigabe weicher Drogen und eine striktere Trennung von Kirche und Staat. ${ }^{30}$ Bei diesen Fragen stehen, abgesehen von kommerziellen Aspekten des Urheberrechtes, kulturelle beziehungsweise mentalitätsbezogene Aspekte im Vordergrund.

Die einzige prominente Aussage zur Sozialpolitik ist die Forderung nach einem bedingungslosen Grundeinkommen. ${ }^{31}$ Dieser Programmpunkt könnte als Indiz dafür gewertet werden, dass als Grundlage für den Zuspruch für die Piratenpartei auch ein Konflikt in Frage kommt, bei dem es auf die Verteilung des gesellschaftlichen Wohlstandes ankommt.

24 Vgl. zum Beispiel Nina Trentmann, Piraten feiern ersten und vielleicht letzten Erfolg, in: Welt online vom 27. September 2009, http://www.welt.de/politik/bundestagswahl/article4647418/ Piraten-feiern-ersten-und-vielleicht-letzten-Erfolg.html (Abruf am 7. August 2012).

$25 \mathrm{Vgl}$. Oskar Niedermayer, Erfolgsbedingungen neuer Parteien im Parteiensystem am Beispiel der Piratenpartei Deutschland, in: ZParl, 41. Jg. (2010), H. 4, S. 838 - 854, S. 839.

26 Vgl. Gero Neugebauer/ Richard Stöss, a.a.O. (Fn. 16), S. 271.

27 Juli Zeh, Augen zu und durch, in: SZ-Magazin vom 25. November 2011, S. 20 - 23. Wie schwierig eine Verortung der Piraten im Verhältnis zu den anderen Parteien ist, wird an folgendem Beispiel deutlich: Im Bezirksparlament des Hamburger Stadtteils Bergedorf bildet die Piratenpartei eine Fraktionsgemeinschaft mit der FDP; im Stadtparlament der Kreisfreien Stadt Oldenburg in Niedersachsen dagegen mit der Linkspartei.

28 Zur Software Liquid Feedback siehe http://liquidfeedback.org/open-source/project/ (Abruf am 7. August).

29 Hannah Beitzer, Piraten erklären, was Piratenpolitik ist, in: süddeutsche.de vom 5. Oktober 2011, http://www.sueddeutsche.de/politik/auftritt-vor-der-bundespressekonferenz-piraten-erklaerenwas-piratenpolitik-ist-1.1155747 (Abruf am 7. August 2012).

30 Vgl. Christoph Bieber, a.a.O. (Fn. 21), S. 29.

31 Vgl. Hannah Beitzer, Grundeinkommen ist nicht links, sondern notwendig, in: süddeutsche.de vom 3. Dezember 2011, http://www.sueddeutsche.de/politik/parteitag-der-piraten-in-offenbachmehrheit-fuer-das-grundeinkommen-1.1225654 (Abruf am 7. August). 
Im Zuge der ökonomischen Liberalisierung der 1990er und 2000er Jahre ist eine Generation beruflich sozialisiert worden, die der Aufforderung, eigenverantwortlich zu handeln, gefolgt ist und auf mannigfaltige Art unter prekären Bedingungen abhängig beschäftigt oder auch selbständig berufstätig ist. Der berufliche Status des Personenkreises, auf den diese Charakterisierung zutrifft, wechselt häufig zwischen Selbständigkeit, abhängiger Beschäftigung und Arbeitslosigkeit. ${ }^{32}$ Das bedingungslose Grundeinkommen könnte in einer weitgehend liberalisierten Ökonomie, die nach zunehmender Individualität und Selbstverantwortung verlangt, die persönliche Autonomie und die individuelle Freiheit schützen, indem das Sicherheitsbedürfnis des Einzelnen befriedigt wird.

Sowohl die genannten kulturellen und mentalitätsbezogenen Gesichtspunkte als auch Aspekte, die die berufliche Situation und die soziale Sicherheit betreffen, deuten darauf hin, dass mit dem sozialen Wandel Generationenkonflikte einhergehen können. Dies gilt möglicherweise auch für die Auffassungen von Geschlechterrollen, die einer eigenen Dynamik unterworfen sind. Der Umstand, dass die Mitglieder und die Wähler der Piratenpartei sehr jung sind ${ }^{33}$, legt die Interpretation nahe, dass ein sozialer oder kultureller Konflikt vorliegen könnte, der mit einem Generationenkonflikt einher geht. Demnach sollte die Deutung spezifischer Konfliktlagen nicht nur auf der Betrachtung generationen- und geschlechtsspezifischen Wahlverhaltens beruhen ${ }^{34}$, sondern darauf, wie alters- und geschlechtsspezifische Interessenlagen in sozialstrukturelle und kulturelle Konflikte einer sich wandelnden Gesellschaft eingebettet sind.

\section{Empirische Analyse zur Wählerschaft der Piratenpartei}

Bei einer Untersuchung der Anhängerschaft der Piraten stellt sich bisher das Problem zu geringer Fallzahlen ${ }^{35}$, so dass kaum belastbare Aussagen möglich sind. Um dieses Problem so weit wie möglich zu beheben, werden in der folgenden Analyse zwei Datensätze herangezogen, die eine aussagekräftige Anzahl von Piratenwählern enthalten. Zum einen wird auf kumulierte Daten aus Wahlbefragungen von insgesamt sieben Landtagswahlen zurückgegriffen, die im Rahmen der German Longitudinal Election Study (GLES) durchgeführt wurden. ${ }^{36}$ Der zweite Datensatz geht auf eine Erhebung der Abteilung für empirische De-

32 Vgl. Hans J. Pongratz / Stefanie Simon, Prekaritätsrisiken unternehmerischen Handelns, in: Andrea D. Bührmann / Hans J. Pongratz (Hrsg.), Prekäres Unternehmertum: Unsicherheiten von selbstständiger Erwerbstätigkeit und Unternehmensgründung, Wiesbaden 2010, S. 27 - 60.

33 Vgl. Daten der regionalen Erstwählerbefragung in diesem Beitrag.

34 Vgl. Hanna Kaspar / Jürgen W. Falter, Entstehung neuer Konfliktlinien: Geschlechterkonflikt und Alterskonflikt?, in: Hans Rattinger / Oscar W. Gabriel / Jürgen W. Falter (Hrsg.), Der gesamtdeutsche Wähler, Baden-Baden 2007, S. 113 - 140.

35 In der Nachwahlbefragung zur Bundestagswahl 2009 im Rahmen der German Longitudinal Election Study (GLES) wurden beispielsweise lediglich 16 Wähler der Piratenpartei erfasst.

36 Daten der GLES (erhältlich unter http://www.gesis.org/wahlen/gles/daten-und-dokumente/daten) für folgende Landtagswahlen: Nordrhein-Westfalen 2010 (ZA-Nummer: 5324), Baden-Württemberg 2011 (ZA-Nummer 5328), Rheinland-Pfalz 2011 (ZA-Nummer: 5327), Sachsen-Anhalt 2011 (ZA-Nummer: 5325), Mecklenburg-Vorpommern 2011 (ZA-Nummer: 5330) und Berlin 2011 (ZA-Nummer: 5329). N = 3.368, darunter 90 Wähler der Piratenpartei (ungewichteter Datensatz). Daten der Wahlen in Bremen und Hamburg 2011 können aufgrund fehlender Codierung der Piraten nicht einbezogen werden. In die Analyse gehen nur Befragte ein, die zum 
mokratieforschung und der Arbeitsgruppe Didaktik der politischen Bildung der Universität Oldenburg zurück. Die Untersuchung war auf das Abstimmungsverhalten von Erstwählern bei der Bundestagswahl 2009 gerichtet. Damit wurde eine Altersgruppe erfasst, die deutlich überproportional für die Piratenpartei stimmt. ${ }^{37}$

Aus beiden Datensätzen werden zuerst die sozialstrukturellen und soziodemographischen Besonderheiten der Piratenwählerschaft herausgearbeitet. In einem zweiten Schritt erfolgt jeweils eine Analyse der politischen Einstellungen und Mentalitäten dieser Gruppe. Eine gruppenspezifische Homogenität für einen der beiden oder beide Aspekte der Wählerschaft der Piraten könnte als Indiz gewertet werden, dass ein Cleavage für die neue Partei konstitutiv ist.

Um Hinweise zu finden, ob die Piraten eine realistische Chance auf einen Einzug in den Bundestag haben, werden beide Datensätze auch vor dem Hintergrund der Frage betrachtet, welche Schlüsse sich für das Wählerpotenzial ergeben. Als eine Reflexionsfläche für vorsichtige Aussagen über dieses Potenzial wird eine Nachwahlbefragung von Infratest Dimap herangezogen, die nach der Wahl zum Berliner Abgeordnetenhaus durchgeführt wurde. ${ }^{38}$

\subsection{Daten der German Longitudinal Election Study}

\section{Soziodemographische und sozioökonomische Charakteristika}

Hinsichtlich des Alters und des Geschlechts ergibt sich ein eindeutiges Bild. Die jungen Kohorten, 18 bis 24 Jahre und 25 bis 34 Jahre, sind in der Wählerschaft der Piraten deutlich überrepräsentiert, der Anteil der beiden mittleren Kohorten ( 35 bis 44 und 45 bis 54 Jahre) in der Piratenwählerschaft nähert sich dem Anteil dieser Altersgruppen in der Gesamtstichprobe an. In den älteren Kohorten ist die Piratenpartei dagegen deutlich unterrepräsentiert. Zudem kann festgehalten werden, dass mehr als drei Viertel ihrer Wähler männlich sind. ${ }^{39}$

Zeitpunkt der Befragung beabsichtigten, ihre Stimme abzugeben oder durch Briefwahl bereits gewählt hatten. Wähler der sonstigen Parteien werden aufgrund der geringen Fallzahlen nicht ausgewiesen. Zudem ist diese Kategorie sehr heterogen, was eine sinnvolle Interpretation unmöglich macht. Durch die Aktivierung der Repräsentativgewichte und Unit Non-Response reduziert sich die tabellierte Fallzahl. Da die Befragung online in einem Befragtenpanel durchgeführt wurde, ist an dieser Stelle auf das Problem der Selbstselektion der Teilnehmer und die daraus resultierende eigeschränkte Repräsentativität hinzuweisen.

37 Die Befragung wurde an insgesamt elf Berufsschulen und Gymnasien im Weser-Ems-Gebiet schriftlich durchgeführt. Die Befragten, die für die Auswertung berücksichtigt wurden, waren zwischen 18 und 25 Jahren alt. Um ein möglichst breites Spektrum der verschiedenen Bildungsgänge abzudecken, wurden Oberstufen an Gymnasien, Berufsbildenden Schulen und Gesamtschulen bei der Befragung berücksichtigt. Der Anteil der Piratenpartei in der Stichprobe $(\mathrm{N}=$ 1.054, davon 108 Nichtwähler und 120 Antwortverweigerer) betrug 9,9 Prozent (81 Befragte). Weitere Erläuterungen über die Jungwählerbefragung finden sich in Fn. 44.

38 Vgl. Viola Neu / Michael Borchard, Abgeordnetenhauswahl in Berlin, Berlin 2011, http://www. kas.de/wf/doc/kas_28849-544-1-30.pdf?111108135728 (Abruf am 7. August 2012).

39 Zahlen von Infratest Dimap aus Berlin zeigen, dass die Piraten auch bei dem gegenüber den vorangegangenen Landtagswahlen deutlich gesteigerten Ergebnis von denselben Gruppen überproportional gewählt werden. Unter den 18- bis 24-jährigen Männern gewinnen sie 20 Prozent und unter den 25- bis 34-jährigen 21 Prozent, im Vergleich zu ihrem Gesamtergebnis von 8,9 Prozent. Bei Frauen sind es in beiden Altersgruppen jeweils zwölf Prozent. Vgl. ebenda. 


\begin{tabular}{|c|c|c|c|c|c|c|c|}
\hline & Gesamt & Piraten & $\mathrm{CDU}$ & SPD & FDP & Grüne & Linke \\
\hline \multicolumn{8}{|l|}{ Altersgruppen } \\
\hline 18 bis 24 & 6,7 & 15,5 & 6,4 & 6,1 & 6,4 & 11,4 & 3,2 \\
\hline 25 bis 34 & 14,5 & 29,6 & 13,6 & 13,4 & 22,0 & 15,8 & 10,7 \\
\hline 35 bis 44 & 14,5 & 19,7 & 15,0 & 11,8 & 23,9 & 17,9 & 12,7 \\
\hline 45 bis 54 & 20,6 & 15,5 & 18,8 & 19,5 & 21,1 & 21,4 & 24,1 \\
\hline 55 bis 64 & 26,0 & 12,7 & 21,4 & 28,4 & 17,4 & 21,1 & 35,8 \\
\hline 65 und älter & 17,7 & 7,0 & 24,6 & 20,7 & 9,2 & 12,3 & 13,5 \\
\hline $\mathrm{N}$ & 2.345 & 71 & 499 & 701 & 109 & 341 & 497 \\
\hline \multicolumn{8}{|l|}{ Geschlecht } \\
\hline Männlich & 55,0 & 76,4 & 54,7 & 54,8 & 56,0 & 44,0 & 56,7 \\
\hline Weiblich & 45,0 & 23,6 & 45,3 & 45,2 & 44,0 & 56,0 & 43,3 \\
\hline $\mathrm{N}$ & 2.346 & 72 & 499 & 701 & 109 & 341 & 497 \\
\hline \multicolumn{8}{|l|}{ Schulabschluss } \\
\hline Kein Abschluss / Hauptschule & 35,5 & 12,5 & 31,7 & 42,5 & 30,9 & 31,4 & 34,8 \\
\hline Realschule & 33,5 & 30,6 & 35,1 & 30,5 & 34,5 & 27,0 & 39,2 \\
\hline Abitur & 30,8 & 55,6 & 32,9 & 26,7 & 34,5 & 40,8 & 26,0 \\
\hline $\mathrm{N}$ & 2.346 & 72 & 498 & 701 & 110 & 341 & 497 \\
\hline \multicolumn{8}{|l|}{ Beschäftigungsstatus } \\
\hline Berufstätig & 50,8 & 54,8 & 49,0 & 45,8 & 72,2 & 58,3 & 49,3 \\
\hline Ausbildung & 6,6 & 15,1 & 5,6 & 6,4 & 5,6 & 12,2 & 4,3 \\
\hline Arbeitslos / Umschulung & 8,1 & 13,7 & 4,6 & 6,7 & 5,6 & 7,4 & 13,6 \\
\hline Rente & 29,7 & 11,0 & 36,3 & 35,8 & 12,0 & 17,9 & 28,4 \\
\hline Nicht berufstätig & 4,7 & 5,5 & 4,4 & 5,3 & 4,6 & 4,2 & 4,5 \\
\hline $\mathrm{N}$ & 2.333 & 73 & 498 & 699 & 108 & 336 & 493 \\
\hline \multicolumn{8}{|l|}{ Beschäftigungssektor } \\
\hline Öffentlicher Dienst & 27 & 15,3 & 29,3 & 19 & 17,9 & 26,9 & 25,8 \\
\hline Gemeinnütziges Unternehmen & 9,3 & 15,4 & 5,4 & 8,1 & 5,7 & 16,2 & 10,4 \\
\hline Privatwirtschaftliches Unternehmen & 63,7 & 69,2 & 65,2 & 62,9 & 76,4 & 57,0 & 63,8 \\
\hline $\mathrm{N}$ & 2.209 & 65 & 460 & 669 & 106 & 309 & 480 \\
\hline \multicolumn{8}{|l|}{ Beschäftigungsverhältnis } \\
\hline Selbständige / Freiberufler & 11,4 & 12,3 & 16,2 & 7,4 & 17,1 & 13,1 & 11,1 \\
\hline Beamte & 6,5 & 3,1 & 9,7 & 7,9 & 2,9 & 7,0 & 2,9 \\
\hline Angestellte & 61,0 & 69,2 & 59,6 & 61,0 & 61,9 & 62,9 & 59,3 \\
\hline Arbeiter & 18,3 & 9,2 & 10,5 & 21,0 & 15,2 & 13,1 & 25,8 \\
\hline Sonstige & 2,9 & 6,2 & 4,0 & 2,7 & 2,9 & 3,8 & 1,0 \\
\hline $\mathrm{N}$ & 2.237 & 65 & 475 & 675 & 105 & 313 & 481 \\
\hline \multicolumn{8}{|l|}{ Einkommen } \\
\hline Bis $900 €$ & 14,5 & 12,7 & 10,6 & 12,1 & 12,4 & 16,1 & 18,8 \\
\hline Bis $1.500 €$ & 24,3 & 15,5 & 20,2 & 24,0 & 12,4 & 26,0 & 30,6 \\
\hline Bis $2.000 €$ & 15,6 & 22,5 & 14,2 & 17,4 & 21,9 & 15,2 & 14,0 \\
\hline Bis $2.600 €$ & 17,9 & 16,9 & 17,5 & 19,5 & 17,1 & 14,6 & 19,4 \\
\hline Bis $3.500 €$ & 13,5 & 15,5 & 17,1 & 13,1 & 17,1 & 12,8 & 9,7 \\
\hline $3.500 €$ und mehr & 14,2 & 16,9 & 20,4 & 13,9 & 19,0 & 15,2 & 7,4 \\
\hline $\mathrm{N}$ & 2.255 & 71 & 480 & 662 & 105 & 335 & 484 \\
\hline
\end{tabular}


Hinzu kommt der hohe Anteil an Anhängern mit Abitur, was mit dem Alter der Piratenwähler korreliert, da die jüngere Generation häufiger formal höhere Bildungsabschlüsse erwirbt.

Die Überrepräsentation junger Anhänger in der Wählerschaft der Piraten kann als neue politische Vertretung einer bisher im politischen Prozess vernachlässigten Gruppe gedeutet werden. Junge Menschen tendieren nach der erstmaligen Beteiligung an Wahlen dazu, in ein Partizipationsloch zu fallen. ${ }^{40}$ Diese Repräsentationslücke könnten die Piraten füllen. Die möglichen Gründe dafür liegen in den von der älteren Generation abweichenden Interessenschwerpunkten, die dieser Lebensphase immanent sind. ${ }^{41}$

Ergänzt werden die bisherigen Ergebnisse durch den Befund, dass die Wähler der Piraten vornehmlich im privatwirtschaftlichen Sektor beschäftigt sind. In dieser Gruppe, die mit 63,7 Prozent den größten Teil der Befragten ausmacht, ist die Piratenpartei etwas überrepräsentiert: 69,2 Prozent ihrer Wähler arbeiten in diesem Sektor. Berufstätige aus gemeinnützigen Unternehmen sind deutlich überrepräsentiert, umgekehrt ist das Verhältnis bei Beschäftigten des öffentlichen Dienstes. Hinsichtlich des Beschäftigungsstatus zeigt sich, dass die Piraten von Rentnern deutlich seltener gewählt werden als von Angehörigen anderer Gruppen. Ein überproportionaler Anteil an überproportionalen Anteil an Piraten-Wählern befindet sich ,in Ausbildung“, da in dieser Gruppe naturgemäß überwiegend junge Wähler zu finden sind. Arbeitslose und Wähler in Umschulungsmaßnahmen tendieren ebenfalls überproportional zu den Piraten; Selbständige und Freiberufler sind etwas überrepräsentiert. Wähler, die der größten Gruppe, den Angestellten, zugeordnet werden (61 Prozent des Samples), neigen den Piraten leicht überdurchschnittlich zu: 69,2 Prozent ihrer Wähler sind Angestellte. Arbeiter und Beamte sind dagegen in der Piraten-Wählerschaft jeweils nur etwa halb so stark vertreten wie unter allen Befragten.

Beim Einkommen sind die Piratenwähler in der Gruppe, die monatlich zwischen 1.500 und 2.000 Euro verdient, am stärksten überrepräsentiert. In den angrenzenden Gruppen ist das Verhältnis dagegen umgekehrt. Die höheren Einkommensschichten sind in der Piratenwählerschaft wiederum etwas stärker vertreten. Im Hinblick auf das Alter und das Geschlecht zeigt sich für die Wählerschaft der Piratenpartei das höchste Ausmaß an Homogenität. Bei den Variablen, die ihre Beschäftigungssituation betreffen, sind Gemeinsamkeiten erkennbar, aber weniger deutlich.

\section{Ideologische Charakteristika und politische Einstellungen}

Bei der klassischen Standardvariablen zeigt sich, dass die Wähler der Piraten im Vergleich zu den Wählern aller anderen Parteien das größte Interesse an Politik angaben. Zugleich sind sie aber mit 46,5 Prozent, im Vergleich zu 33,5 Prozent unter allen Befragten, überproportional häufig unzufrieden mit der Demokratie in Deutschland. Nur in der Wählerschaft der Linken ist der Anteil der Unzufriedenen mit 55,8 Prozent noch höher.

40 Vgl. Simone Abendschön / Sigrid Roßteutscher, Jugend und Politik: Verliert die Demokratie ihren Nachwuchs?, in: Evelyn Bytzek / Sigrid Roßteutscher (Hrsg.), Der unbekannte Wähler? Mythen und Fakten über das Wahlverhalten der Deutschen, Frankfurt am Main 2011, S. 59 - 80.

41 Vgl. Klaus Hurrelmann, Lebensphase Jugend. Eine Einführung in die sozialwissenschaftliche Jugendforschung, Weinheim / München 2009, S. 27. 


\begin{tabular}{|c|c|c|c|c|c|c|c|}
\hline & Gesamt & Piraten & $\mathrm{CDU}$ & SPD & FDP & Grüne & Linke \\
\hline \multicolumn{8}{|l|}{ Politikinteresse } \\
\hline Stark / sehr stark & 52,9 & 61,1 & 53,1 & 50,4 & 51,4 & 52,6 & 53,5 \\
\hline Mittelmäßig & 37,1 & 30,6 & 38,6 & 39,7 & 37,6 & 35,0 & 36,8 \\
\hline Weniger stark / überhaupt nicht & 10,0 & 8,3 & 8,2 & 9,8 & 11,0 & 12,4 & 9,7 \\
\hline $\mathrm{N}$ & 2.343 & 72 & 497 & 702 & 109 & 340 & 497 \\
\hline \multicolumn{8}{|l|}{ Zufriedenheit mit der Demokratie } \\
\hline Ziemlich / sehr zufrieden & 24,8 & 12,7 & 44,5 & 24,4 & 28,0 & 26,8 & 9,5 \\
\hline Teils teils & 41,8 & 40,8 & 43,7 & 47,1 & 43,9 & 42,2 & 34,7 \\
\hline Ziemlich / sehr unzufrieden & 33,5 & 46,5 & 11,8 & 28,5 & 28,0 & 31,0 & 55,8 \\
\hline $\mathrm{N}$ & 2.304 & 71 & 490 & 688 & 107 & 339 & 484 \\
\hline
\end{tabular}

Mithilfe der ideologischen Positionierung kann veranschaulicht werden, wie die Wähler der Piraten im Verhältnis zu den Unterstützern der anderen Parteien zu verorten sind. Hierzu werden drei Items herangezogen: Als erster Indikator dient die Frage, ob der Zuzug von Ausländern erleichtert oder erschwert werden sollte (libertär-autoritäre Dimension). Je höher der Skalenwert, desto stärker wird die Einschränkung der Zuwanderung befürwortet. Als zweiter Indikator wird die Haltung zu Fragen staatlicher Leistungen und der Steuerpolitik herangezogen (sozioökonomische Dimension). Der Wert 1 bedeutet dabei, dass Steuern und Staatsausgaben reduziert, der Wert 11, dass die staatlichen Leistungen und Ausgaben ausgebaut werden sollen. Drittens wurde die klassische Frage nach der Positionierung auf der Links-Rechts-Skala gestellt. Ein Wert von 1 bedeutet dabei „links“, ein Wert von 11 „rechts“.

Aus den Mittelwerten, die sich aus den Orientierungen der Parteiwählerschaften auf den ersten beiden Dimensionen ergeben, wurde ein bivariates Streudiagramm erstellt. Zur besseren Verständlichkeit wurden gepunktete Hilfslinien für den Mittelwert über alle Befragte eingezogen. Das zweite Schaubild zeigt die Positionierung der Parteiwählerschaften auf dem Links-Rechts-Kontinuum.

Im ersten Diagramm sind die Wähler der Piraten tendenziell eher im Spektrum der linken Parteien zu verorten. Auf der libertär-autoritären Dimension ordnen sie sich zwischen der SPD und Bündnis 90/Die Grünen ein, auf der sozioökonomischen Dimension zwischen der SPD und der CDU.

Auf dem univariaten Links-Rechts-Kontinuum positionieren sich die Wähler der Piraten in der Mitte zwischen dem rot-grünen und dem schwarz-gelben Lager. Sie liegen annähernd auf dem Mittelwert der Gesamtstichprobe. Diese Befunde passen in das Bild der bisherigen Ergebnisse: Die Piratenwählerschaft entspringt der sozialen Mitte und orientiert sich in der politischen Mitte. Insgesamt scheinen einstellungsbezogene Kriterien für die Wahlentscheidung zugunsten der Piratenpartei etwas wichtiger zu sein als soziologische Kriterien, mit Ausnahme von Geschlecht und Alter. ${ }^{42}$

42 Die Daten von Infratest dimap zur Wahl des Berliner Abgeordnetenhauses bestätigen die Befunde im Wesentlichen. Den Frauenanteil konnte die Partei jedoch gegenüber der Stichprobe von etwa einem Viertel auf rund ein Drittel steigern. 


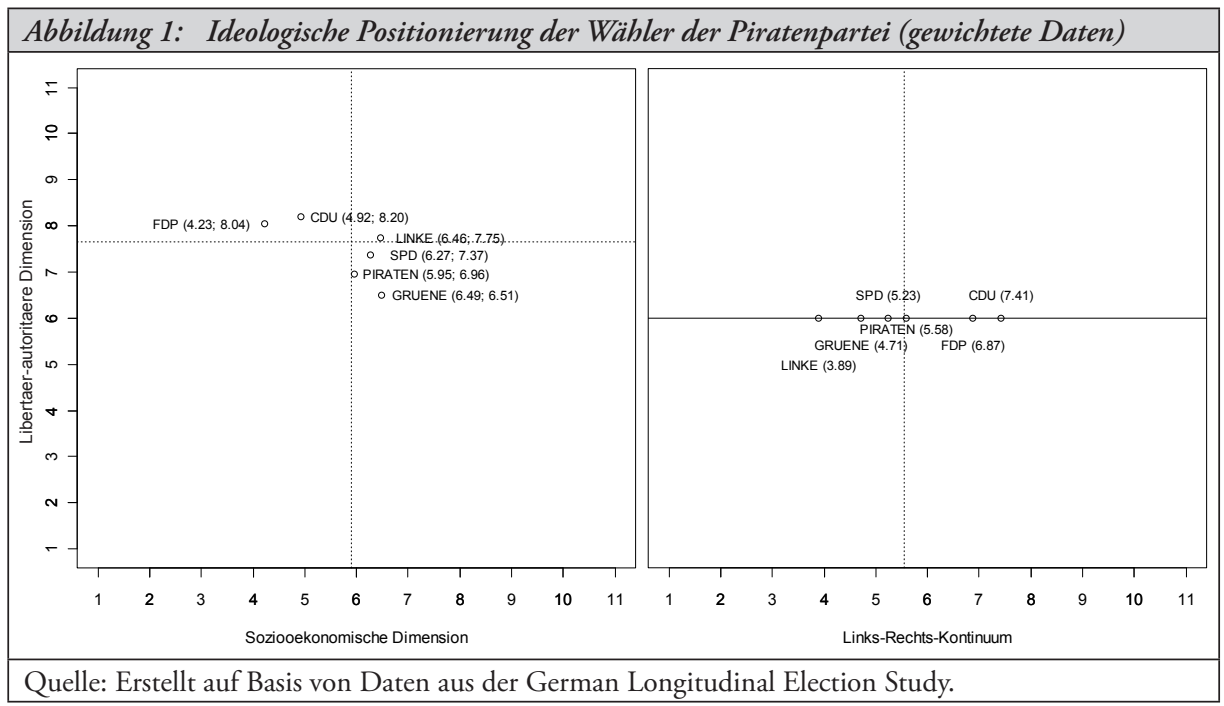

\subsection{Daten der regionalen Erstwählerbefragung}

Die Gruppe der Erstwähler ist für diese Analyse relevant, da die Piraten in dieser Gruppe deutlich überdurchschnittlich abschneiden. Von Bedeutung sind Erstwähler im vorliegenden Kontext zudem, weil die erstmalige Wahlentscheidung für eine Partei einen hohen symbolischen Wert hat und sich verfestigen kann. ${ }^{43}$ Würde sich die Wählerschaft der Piraten in dieser Kohorte hinsichtlich der sozialen Herkunft und der politischen Einstellungen wesentlich von der Gesamtwählerschaft unterscheiden, könnte dies dafür sprechen, dass sich das Wahlverhalten dieser Gruppe verstetigt.

Aufgrund der Vorgehensweise bei der Erhebung kann keine vollständige Repräsentativität beansprucht werden. Ein Vergleich mit der repräsentativen Wahlstatistik zeigt aber, dass die Stichprobe durchaus aussagekräftige Ergebnisse liefert. ${ }^{44}$ Ein Blick auf das geschlechts-

43 Wie stark der Kohorteneffekt das Wahlverhalten im Lebenslauf bestimmt, ist jedoch umstritten. Vgl. Rolf Becker / Anja Mays, Soziale Herkunft, politische Sozialisation und Wählen im Lebensverlauf, in: PVS, 44. Jg. (2003), H. 1, S. $19-40$.

44 Auf der Website des Bundeswahlleiters sind die Ergebnisse der Altersgruppe der 18- bis 25-Jährigen für alle Bundesländer separat ausgewiesen, http://www.bundeswahlleiter.de/de/bundestagswahlen/ BTW_BUND_09/veroeffentlichungen/repraesentative/index.html (Abruf am 7. August 2012). Ein Vergleich der Stichprobe mit dem Abstimmungsverhalten dieser Altersgruppe in Niedersachsen zeigt, dass die Grünen etwas überrepräsentiert (Stichprobenanteil 20,5 Prozent, Zweitstimmenanteil 15,4 Prozent) und die Linken etwas unterrepräsentiert sind (6,9 gegenüber 9,0 Prozent). Der hohe Stimmenanteil für die Piratenpartei in der Stichprobe (9,9 Prozent, 81 Wähler) und der „Sonstigen“ (2,6 Prozent) korrespondiert mit dem Ergebnis der sonstigen Parteien, die in der amtlichen Statistik nicht einzeln ausgewiesen werden (zusammen 13,9 Prozent). Bei der CDU (21,6 Prozent in der Stichprobe und 24,2 Prozent in Niedersachsen) und der SPD (25,0 und 23,5 Prozent) sind die Unterschiede im Vergleich zum niedersächsischen Landesergebnis gering. Die vorhandenen Abweichungen der Untersuchungsergebnisse von den Ergebnissen der repräsentativen Wahlstatistik lassen sich dadurch erklären, dass einige Gruppen (zum Beispiel arbeitslose Jugendliche) mit der angewendeten Erhebungsmethode nicht erreicht werden konnten. Hinzu kommen regionale Besonderheiten innerhalb des Erhebungsgebietes, das nur Teile Niedersachsens umfasste (vgl. auch Fn. 37). 
spezifische Wahlverhalten bestätigt die bisherigen Ergebnisse: In der Stichprobe sind mehr als drei Viertel der Piratenwähler männlich.

\section{Schichtspezifische Hintergründe}

Unter den Erstwählern der Piraten sind überdurchschnittlich viele Personen zu finden, die sich in einer Berufsausbildung befinden beziehungsweise eine solche anstreben, darunter viele Gymnasiasten.

\begin{tabular}{|c|c|c|c|c|c|c|c|c|}
\hline & Gesamt & Piraten & $\mathrm{CDU}$ & SPD & FDP & Grüne & Linke & N.-W. \\
\hline \multicolumn{9}{|l|}{$\begin{array}{l}\text { Angestrebter } \\
\text { Ausbildungsabschluss* }\end{array}$} \\
\hline Berufsausbildung & 60,1 & 67,6 & 60,6 & 59,2 & 64,2 & 43,7 & 58,0 & 73,7 \\
\hline Hochschulstudium & 37,7 & 29,6 & 35,5 & 40,2 & 34,7 & 53,3 & 40,0 & 25,3 \\
\hline $\mathrm{N}$ & 794 & 71 & 155 & 174 & 95 & 135 & 50 & 95 \\
\hline \multicolumn{9}{|l|}{$\begin{array}{l}\text { Haushaltsnettoeinkommen } \\
\text { des Elternhauses der } \\
\text { Parteiwählerschaften }\end{array}$} \\
\hline Unter $1.000 €$ & 6,2 & 5,5 & 1,6 & 3,3 & 4,8 & 9,3 & 20,5 & 12,2 \\
\hline 1.000 bis unter $2.000 €$ & 28,8 & 34,5 & 28,0 & 28,5 & 25,3 & 32,2 & 27,3 & 30,9 \\
\hline 2.000 bis unter $3.000 €$ & 31,2 & 25,5 & 26,4 & 37,7 & 31,3 & 27,1 & 27,3 & 32,5 \\
\hline $3.000 €$ und mehr & 33,8 & 34,5 & 44,0 & 30,5 & 38,6 & 31,4 & 25,0 & 24,4 \\
\hline $\mathrm{N}$ & 662 & 55 & 125 & 151 & 83 & 118 & 44 & 74 \\
\hline \multicolumn{9}{|l|}{ Subjektive Schichteinstufung } \\
\hline $\begin{array}{l}\text { Unterschicht / untere Mittel- } \\
\text { schicht }\end{array}$ & 13,7 & 17,8 & 10,9 & 13,1 & 12,7 & 13,8 & 28,3 & 14,3 \\
\hline Mittelschicht & 58,1 & 64,4 & 50,0 & 67,9 & 46,1 & 55,3 & 60,4 & 61,9 \\
\hline $\begin{array}{l}\text { Obere Mittelschicht / Ober- } \\
\text { schicht }\end{array}$ & 27,2 & 17,8 & 39,1 & 18,9 & 41,1 & 31,0 & 11,3 & 23,8 \\
\hline $\mathrm{N}$ & 831 & 73 & 156 & 190 & 102 & 152 & 53 & 90 \\
\hline \multicolumn{9}{|l|}{$\begin{array}{l}\text { Beruflicher Hintergrund } \\
\text { des Elternhauses }\end{array}$} \\
\hline Arbeiterhaushalt & 28,0 & 30,4 & 43,6 & 23,7 & 20,4 & 16,8 & 36,4 & 33,3 \\
\hline Selbstständige & 20,7 & 11,6 & 10,6 & 32,9 & 30,1 & 21,7 & 11,4 & 15,3 \\
\hline Beamtenhaushalt & 16,1 & 17,4 & 11,7 & 15,8 & 15,1 & 18,9 & 13,6 & 18,1 \\
\hline Angestellte & 33,2 & 37,7 & 33,5 & 26,3 & 32,3 & 39,9 & 34,1 & 31,1 \\
\hline $\mathrm{N}$ & 728 & 69 & 152 & 179 & 93 & 143 & 44 & 88 \\
\hline
\end{tabular}

Anmerkung: N.-W. = Nichtwähler; * beim Ausbildungsabschluss wird die Kategorie „Sonstige“ nicht tabelliert. Die Kategorie „weiß nicht“ wird als fehlender Wert von der Analyse ausgeschlossen.

Quelle: Eigene Erhebung (siehe Fn. 37).

Hinsichtlich der Einkommenssituation des Elternhauses gibt es keine gravierenden Abweichungen vom Gesamtergebnis. Das gilt auch für den beruflichen Hintergrund des Elternhauses, mit Ausnahme der Jungwähler aus Selbständigenhaushalten, die in der Piratenwählerschaft deutlich unterrepräsentiert sind. Bei der subjektiven Schichteinstufung fällt der überdurchschnittliche Anteil auf, der sich der Mittelschicht zuordnet (64,4 Prozent im Ver- 
gleich zu 58,1 Prozent von allen Befragten). In den höheren Schichten verorten sich die Wähler der Piratenpartei dagegen deutlich unterdurchschnittlich. Sie kommen auch relativ selten aus Selbständigenhaushalten; die übrigen Gruppen sind in ihrer Wählerschaft jeweils geringfügig überrepräsentiert.

\section{Wertvorstellungen und politische Einstellungen}

Hinsichtlich des Politikinteresses und der Demokratiezufriedenheit zeigt sich für die Erstwähler der Piraten derselbe Zusammenhang wie in dem Datensatz der GLES: Sie sind überdurchschnittlich häufig an Politik interessiert, aber seltener zufrieden mit der Demokratie als die Wähler der anderen Parteien mit Ausnahme der Linkswähler und der Nichtwähler.

\begin{tabular}{|l|r|r|r|r|r|r|r|r|}
\hline \multicolumn{10}{|c|}{ Tabelle 4: Politikinteresse und Demokratiezufriedenheit der befragten Erstwähler (in Prozent) } \\
\hline & $\begin{array}{r}\text { Ge- } \\
\text { samt }\end{array}$ & Piraten & CDU & SPD & FDP & Grüne & Linke & N.-W. \\
\hline Politikinteresse & & & & & & & & \\
Stark / sehr stark & 38,4 & 53,1 & 35,2 & 40,7 & 38,0 & 41,2 & 30,4 & 9,0 \\
Mittelmäßig & 43,2 & 26,6 & 50,0 & 41,7 & 40,7 & 47,3 & 48,2 & 47,3 \\
Weniger stark / gar nicht & 18,4 & 20,3 & 14,8 & 17,6 & 12,3 & 11,5 & 19,4 & 45,7 \\
N & 910 & 79 & 176 & 199 & 108 & 165 & 56 & 106 \\
\hline Demokratiezufriedenheit & & & & & & & & \\
Gar nicht/weniger Zufrieden & 26,1 & 34,2 & 13,4 & 21,6 & 16,2 & 29,2 & 52,8 & 36,3 \\
Mäßig zufrieden & 45,2 & 49,4 & 49,7 & 43,3 & 46,7 & 38,5 & 32,1 & 47,7 \\
Sehr zufrieden & 28,7 & 16,4 & 36,8 & 35,0 & 37,2 & 32,3 & 15,1 & 16,0 \\
N & 881 & 79 & 171 & 194 & 105 & 161 & 53 & 98 \\
\hline Anmerkung: N.-W. = Nichtwähler. \\
Quelle: Eigene Erhebung (siehe Fn. 37).
\end{tabular}

Die Wähler der Piraten bilden damit ein politisch interessiertes und gemäßigtes Protestpotential. Denkbar ist, dass diese Wähler aufgrund der innovativen Struktur der Parteiorganisation über das Internet Möglichkeiten der Partizipation sehen, die andere Parteien nicht bieten. Hier sind die Ähnlichkeiten zu den basisdemokratischen Ideen der Grünen in ihrer Gründungsphase kaum zu übersehen - nur dass damals das Internet nicht zur Verfügung stand.

Mit den individuellen Werthaltungen wurde in der Erstwählerbefragung ein weiterer wichtiger Bestimmungsfaktor des Wahlverhaltens erhoben. ${ }^{45}$ Dabei zeigen sich deutliche Unterschiede zwischen den Präferenzen der Wählerschaften. Bei den Piratenwählern gibt es insbesondere bei zwei der fünf aufgeführten Wertvorstellungen Prozentwerte, die deutlich von der Verteilung in der Gesamtstichprobe abweichen. „Persönliche Freiheit“ wird von über 90 Prozent der Piratenwähler genannt, im Vergleich zu rund 55 Prozent in der Gesamtstichprobe. Die Familie ist für sie dagegen von nur sehr nachrangiger Bedeutung, und Umweltschutz wird ebenfalls deutlich unterdurchschnittlich genannt. In dieser Frage grenzen

45 Vgl. Hanspeter Kriesi, Restructuration of Partisan Politics and the Emergence of a New Cleavage Based on Values, in: West European Politics, 33. Jg. (2010), H. 3, S. 673 - 685. 
sich die Wähler der Piraten deutlich von den Grünen ab. Soziale Gerechtigkeit wird von den Piratenwählern als wichtiger Wert etwa genauso häufig genannt wie in der Gesamtstichprobe.

\begin{tabular}{|c|c|c|c|c|c|c|c|c|}
\hline & Gesamt & Piraten & $\mathrm{CDU}$ & SPD & FDP & Grüne & Linke & N.-W. \\
\hline \multicolumn{9}{|l|}{$\begin{array}{l}\text { Wertvorstellungen (bis zu zwei } \\
\text { Nennungen möglich) }\end{array}$} \\
\hline Soziale Gerechtigkeit & 63,4 & 59,5 & 50,6 & 75,9 & 43,0 & 72,3 & 77,8 & 60,0 \\
\hline Persönliche Freiheit & 55,4 & 92,4 & 57,6 & 42,6 & 71,0 & 44,5 & 61,1 & 46,9 \\
\hline Familie & 34,5 & 5,1 & 42,4 & 37,9 & 34,6 & 25,8 & 37,0 & 49,0 \\
\hline Umweltschutz & 18,5 & 10,1 & 6,5 & 21,0 & 9,3 & 43,9 & 9,3 & 12,3 \\
\hline Innere Sicherheit & 19,7 & 22,8 & 33,5 & 16,4 & 25,2 & 7,7 & 9,3 & 17,3 \\
\hline $\mathrm{N}$ & 879 & 79 & 170 & 195 & 107 & 155 & 54 & 98 \\
\hline \multicolumn{9}{|l|}{$\begin{array}{l}\text { Politikpräferenzen (bis zu drei } \\
\text { Nennungen möglich) }\end{array}$} \\
\hline $\begin{array}{l}\text { Mehr Geld in Bildung } \\
\text { investieren }\end{array}$ & 57,0 & 60,3 & 49,1 & 60,5 & 59,1 & 69,3 & 51,8 & 47,5 \\
\hline $\begin{array}{l}\text { Steuern senken, um die } \\
\text { Wirtschaft anzukurbeln }\end{array}$ & 33,4 & 20,5 & 36,4 & 30,0 & 50,0 & 19,9 & 42,9 & 46,5 \\
\hline Mindestlöhne einführen & 26,5 & 24,4 & 15,0 & 37,5 & 10,9 & 28,9 & 44,6 & 27,3 \\
\hline Den Klimaschutz stärken & 24,8 & 12,8 & 19,7 & 27,5 & 20,0 & 42,2 & 14,3 & 15,2 \\
\hline $\begin{array}{l}\text { Zuwanderung stärker } \\
\text { begrenzen }\end{array}$ & 24,0 & 28,2 & 37,6 & 17,0 & 28,2 & 10,8 & 17,9 & 30,3 \\
\hline $\begin{array}{l}\text { Managergehälter gesetzlich } \\
\text { begrenzen }\end{array}$ & 21,2 & 23,1 & 17,9 & 22,5 & 20,9 & 20,5 & 30,4 & 16,2 \\
\hline Atomausstieg fortsetzen & 20,0 & 14,1 & 7,5 & 28,0 & 7,3 & 42,8 & 8,9 & 12,1 \\
\hline $\begin{array}{l}\text { Kriminalität stärker } \\
\text { bekämpfen }\end{array}$ & 16,3 & 14,1 & 27,2 & 11,5 & 15,5 & 9,0 & 16,1 & 22,2 \\
\hline $\begin{array}{l}\text { Deutsche Soldaten } \\
\text { aus Afghanistan abziehen }\end{array}$ & 15,9 & 28,2 & 8,1 & 15,5 & 11,8 & 16,9 & 26,8 & 15,9 \\
\hline Bürokratieabbau & 13,4 & 28,2 & 17,3 & 10,5 & 18,2 & 9,6 & 7,1 & 8,1 \\
\hline Haushaltssanierung & 11,7 & 10,3 & 17,9 & 14,0 & 15,5 & 7,8 & 1,8 & 7,1 \\
\hline $\mathrm{N}$ & 904 & 78 & 173 & 200 & 110 & 166 & 56 & 99 \\
\hline
\end{tabular}

Anmerkung: N.-W. = Nichtwähler; fett $=$ Wertvorstellungen der Piratenwähler, die deutlich von der Verteilung in der Gesamtstichprobe abweichen.

Quelle: Eigene Erhebung (siehe Fn. 37).

Welchen politischen Sachfragen die Erstwähler Priorität beimessen, ist weitgehend konsistent mit ihren Wertvorstellungen. Bei Gerechtigkeitsthemen wie der Frage nach einem Mindestlohn oder der Begrenzung von Managergehältern sind die Wähler der Piraten nahe an der durchschnittlichen Verteilung. Steuersenkungen werden dagegen deutlich unterproportional gefordert. Umweltthemen wie der Atomausstieg und der Klimaschutz werden ebenfalls nur selten genannt. Wichtig ist den Piratenwählern dagegen der Abbau von Bürokratie. Dies stellt ein klassisches Politikziel der liberal-libertären Denkweise dar. Deutlich ist zudem die Zustimmung zum Abzug der Bundeswehr aus Afghanistan. Die Piraten übertreffen in dieser Frage sogar die Anhängerschaft der Linken, die als einzige Partei den Einsatz eindeutig ab- 
lehnt. Aus den Befunden zur Außen- und Migrationspolitik könnte man schließen, dass die Jungwähler der Piraten sich eine zurückhaltende Rolle Deutschlands in der Welt wünschen; es kann auch eine gewisse Skepsis gegenüber der Europäischen Union abgelesen werden.

\section{Fazit: Überwiegend gute Aussichten zum Entern ${ }^{46}$}

Ausweislich der vorgelegten Daten vertritt die Piratenpartei im Hinblick auf verschiedene Kriterien eine homogene Wählergruppe. Neben den häufig diskutierten Merkmalen - die Piratenwähler sind jung und männlich - zeigt sich, dass sie im privaten Sektor arbeiten und bei ihrer Ausbildung häufig eine berufspraktische Orientierung im Blick haben. Die Piratenwähler verorten sich selbst überproportional häufig in der Mittelschicht und lassen sich dieser auch soziologisch zuordnen. ${ }^{47}$ Politisch-ideologisch können sie nicht eindeutig in einem Lager verortet werden, scheinen aber den Wählern von SPD und Grünen politisch etwas näher zu stehen als denen der CDU und der FDP. Die Wähler der Piraten sind politisch sehr interessiert, aber zugleich mit der Demokratie unzufrieden. Für alle anderen Parteien ist bei einem Vergleich des politischen Interesses und der Demokratiezufriedenheit ein umgekehrter Zusammenhang zu beobachten: je stärker das Interesse für Politik desto höher die Zufriedenheit mit der Demokratie.

Als vom konventionellen Politik- und Parteienbetrieb frustrierte Gruppe, die nun über eine neue Partei den Weg in die Parlamente sucht, sind die Parallelen zwischen der Piratenpartei und den frühen Grünen zu Beginn der 1980er Jahre unverkennbar. ${ }^{48}$ Neben einer gemäßigten Protesthaltung zeigt sich eine stark individualistische Orientierung. Dennoch ist der Stellenwert der sozialen Gerechtigkeit für die Piratenwähler etwa durchschnittlich, was die Wähler der Piraten von denen der FDP und der Linken unterscheidet, die in dieser Frage polarisiert sind. Von den Anhängern der Grünen grenzen sich die Piraten vor allem durch die relativ geringe Bedeutung, die sie umweltpolitischen Themen zusprechen, aber auch durch das Geschlecht und die Orientierung auf den privatwirtschaftlichen Beschäftigungssektor $\mathrm{ab}$.

46 Wir danken Hiltrud Naßmacher für wertvolle Anmerkungen und Kommentare.

47 Wobei im Hinblick auf eine solche Zuordnung durchaus Kritik an den herkömmlichen Kategorien der Wahlsoziologie angebracht zu sein scheint. Beispielsweise fallen ein Zahnarzt mit einem Jahreseinkommen von 120.000 Euro und ein freiberuflich tätiger Journalist, der nur 12.000 Euro verdient in dieselbe Kategorie. Die politischen Interessen und die Erwartungen dieser Personen an die Parteien dürften aber auseinanderfallen. Vgl. Hans J. Pongratz / Stefanie Simon, a.a.O. (Fn. 32). In den Kategorien „Angestellte“ und „Arbeiter“ sind vergleichbare Entwicklungen zu beobachten. Die als im Vergleich zu den traditionellen Normalarbeitsverhältnissen häufig als prekär angesehenen Beschäftigungsverhältnisse, die seit den 1990er Jahren eine maßgebliche Bedeutung für den Arbeitsmarkt haben, haben im Hinblick auf die Lebensplanung und Interessenlagen sehr unterschiedliche Folgen. Entsprechend gibt es auch sehr unterschiedliche Auswirkungen auf die politische Orientierung. Vgl. Klaus Dörre, Prekäre Arbeit und soziale Desintegration, in: APuZ, 56. Jg. (2006), H. 40/41, S. 27 - 32, S. 28 f.

48 Siehe zum Beispiel Ferdinand Müller-Rommel, Social Movements and the Greens: New Internal Politics in Germany, in: European Journal of Political Research, 13. Jg. (1985), H. 1, S. 53 - 67; Thomas Poguntke, The Organization of a Participatory Party - the German Greens, in: European Journal of Political Research, 15. Jg. (1987), H. 6, S. 609 - 633; Silke Mende, „Nicht links, nicht rechts, sondern vorn": Ein Geschichte der Gründungsgrünen, München 2011. 
Es lassen sich also deutliche soziodemographische und wertebezogene Unterschiede zu den Wählern der anderen kleineren Parteien nachweisen, was dafür spricht, dass die Piraten ein gesellschaftliches Cleavage repräsentieren. Dieser Konflikt weist allerdings nicht alle Charakteristika einer der historischen Spaltungen auf, sondern trägt lediglich Züge davon. Zudem spricht einiges dafür, dass dieser politische Gegensatz weniger stabil ist als die traditionellen Cleavages. Aufgrund des beschleunigten gesellschaftlichen Wandels ist die Vermittlung zwischen Gesellschaft und politischem System durch die Parteien insgesamt stärker situativ und punktuell sowie fragiler als in den ersten vier Jahrzehnten der alten Bundesrepublik.

Das Internet spielt für die Piratenpartei eine sehr bedeutende Rolle, insbesondere als Symbol für die Wähler, aber auch in der politischen Praxis, um das Bedürfnis der Mitglieder, politische Prozesse mitzubestimmen, zu befriedigen beziehungsweise diesen Eindruck zu vermitteln. Damit kann das Internet als Metapher angesehen werden, gleichsam als Ersatz für die weitgehend zerfallenen Vorfeldorganisationen der historischen Parteien gelten. Die Piratenpartei auf das Internet zu reduzieren würde aber zu kurz greifen; sie könnte - insbesondere angesichts der aktuellen Debatte um den schlechten Zustand der FDP - als neue sozialliberale Kraft betrachtet werden, die sich noch in einem programmatischen Selbstfindungsprozess befindet. Unter Berücksichtigung der Integration des technischen Fortschritts in die Parteiorganisation und die Werthaltung ihrer Wählerschaft spricht vieles für die Hypothese, dass die Piraten eine „Freiheitspartei der Informationsgesellschaft “ 49 sind. Die hochgradig individualistischen Wertvorstellungen der im Zeitalter der Informationstechnologie, der Globalisierung und der gesellschaftlichen Ökonomisierung sozialisierten Piratenwähler, die bisher politisch kaum gebunden waren, haben offenbar ihre „politische Agentur" gefunden. Ob es sich bei den beobachteten Dispositionen und politischen Einstellungen um Lebensphaseneffekte handelt oder ob sich diese verstetigen, ist noch nicht abschließend zu beantworten. Durch die beständigen Umfrageergebnisse über fünf Prozent deutet sich die Möglichkeit an, dass die Piratenpartei bei der nächsten Bundestagswahl ihr Potenzial mobilisieren könnte. Der Erfolg bei der Wahl zum Berliner Abgeordnetenhaus scheint an viele potenzielle Wähler das Signal ausgesendet zu haben, dass die Piraten die Repräsentationshürde des deutschen Wahlsystems überwinden können. Hierfür sprechen der Einzug der Partei in vier Landtage im Jahr 2012 und der sprunghafte Anstieg in den Umfragen.

Sicher ist der Einzug in den Bundestag jedoch keineswegs. Der weitere Erfolg der Piratenpartei hängt naturgemäß auch von der künftigen politischen und wirtschaftlichen Entwicklung in Europa ab, also von Faktoren, auf die die Partei praktisch keinen Einfluss hat. Eine spürbare Verschärfung der Krise um den Euro und das Auftreten wichtiger Fragen der Wohlstandsverteilung könnte die originären Themen der Piraten in den Hintergrund drängen und ihren Wahlchancen schaden. Auch solche Wähler, die ein stark individualistisch geprägtes Freiheitsbedürfnis entwickelt haben, könnten sich in einer entsprechenden Situation Parteien zuwenden, die eher für soziale Sicherheit und politische Stabilität stehen als die Piraten. Ohne entsprechende Schocks sind ihre Aussichten aber relativ vielversprechend. Die gesellschaftliche Tribalisierung scheint sich im Parteiensystem fortzusetzen.

49 Juli Zeh, a.a.O. (Fn. 27). 